\title{
Spectrum and atmosphere models of irradiated transiting giant planets
}

\author{
Ivan Hubeny ${ }^{1}$ and Adam Burrows ${ }^{2}$ \\ ${ }^{1}$ Steward Observatory and Dept. of Astronomy, University of Arizona, \\ 933 N. Cherry Ave., Tucson, AZ 85721, USA \\ email: hubeny@as.arizona.edu \\ ${ }^{2}$ Dept. of Astrophysical Sciences, Princeton University, \\ Peyton Hall, Princeton, NJ 0854, USA \\ email: burrows@astro.princeton.edu
}

\begin{abstract}
We show that a consistent fit to observed secondary eclipse data for several strongly irradiated transiting planets demands a temperature inversion (stratosphere) at altitude. Such a thermal inversion significantly influences the planet/star contrast ratios at the secondary eclipse, their wavelength dependences, and, importantly, the day-night flux contrast during a planetary orbit. The presence of the thermal inversion/stratosphere seems to roughly correlate with the stellar flux at the planet. Such temperature inversions might be caused by an upper-atmosphere absorber whose exact nature is still uncertain.
\end{abstract}

\section{Introduction}

Theoretical modeling of atmospheres of extrasolar giant planets is a young field, about one decade old, and as youngsters usually are, it is very active, restless, and sometimes quite unpredictable. It had recently undergone a transition from a purely care-free stage (no observations were available) to a more difficult stage where there already are some observations to be fit by the theory.

In this paper, we briefly describe our recent efforts in this area. We will try to convince the reader that despite its young age and many shortcomings, the theory is actually doing quite well.

\section{Modeling procedure}

The basic aim of a model atmosphere is to predict the structure (physical and chemical state) of an atmosphere as a function of depth, as well as the theoretical emergent spectrum. The latter is the most important result of a modeling exercise because by comparing the model predictions to actual observations, one might constrain the physical conditions in planet atmospheres, and indirectly also of the whole planet.

The general problem of atmospheres of extrasolar giant planets is an extremely complex one that should generally involve a 3 -dimensional radiation hydrodynamical treatment of atmospheric motions and day/night-side energy redistribution, with departures from chemical equilibrium, and a self-consistent description of clouds (particle size distribution, cloud position in the atmosphere), together with a self-consistent treatment of the radiation field. Such a challenge is still far beyond current computational capabilities, not to mention the lack of, or inadequacies in, many ingredients needed for the modeling effort, such as complete and reliable atomic and molecular data and optical constants for cloud particles, to name just a few. 
Obviously, one has to resort to approximations. It is fair to say that the work of different groups differs mostly by what actual approximation are adopted, and what simplifications are being made. In the absence of exact calculations, it is difficult to decide which approximations are the most restrictive. Therefore, the current and future development of this field will proceed by gradually relaxing more and more approximations, and the observational tests, whenever available, become the judge of whether one is on track.

The current standard model atmospheres of extrasolar planets are based on the following assumptions: (i) the atmosphere is assumed to be a plane-parallel, horizontally homogeneous layer (which reduces the problem to 1-D); (ii) the atmosphere is in hydrostatic equilibrium; (iii) the atmosphere is in radiative equilibrium (or radiative+convective equilibrium in convectively unstable layers); (iv) local thermodynamic equilibrium (LTE) holds (therefore the opacity and emissivity are known functions of only temperature and density, and can thus be pre-tabulated rather than computed on the fly during model construction); and (v) the atmosphere is in a modified chemical equilibrium (the local chemical equilibrium which takes into account possible rainout and consequent depletion of species).

Most groups that produce giant planet model atmospheres adopt the above assumptions, and use different modeling codes (e.g., Seager \& Sasselov 1998, Barman et al. 2001).

Our group has originally used a code described in Burrows et al. (1997), with an additional algorithm to treat radiation scattering in clouds - Sudarsky et al. (2000). We later switched to a more powerful procedure based on adapting the general-purpose stellar atmosphere code TLUSTY (Hubeny 1988; Hubeny \& Lanz 1995), originally designed for non-LTE model atmospheres of hot stars, and extended to other stellar types and accretion disks. The specific variant for the atmospheres of giant planets and brown dwarfs is called CoolTLUSTY, and has been described briefly in Sudarsky et al. (2003), Hubeny et al. (2003), and Burrows et al. (2008). The code uses the above mentioned five basic assumptions. The chemical equilibrium and departures from it due to the rainout of the species is treated following Burrows \& Sharp (1999), and the opacity tables are computed as described in Sharp \& Burrows (2007).

\section{Model bifurcation; existence of stratospheres}

Soon after the Sudarsky et al. (2003) grid of giant planet model atmospheres was completed, the very close-in planet, OGLE-TR-56b, (Konacki et al. 2003) was discovered, with a planet-star separation of a mere $0.0225 \mathrm{AU}$. We set out to extend our grid to higher irradiations, and found that the computed atmospheric structure depends very sensitively on whether the $\mathrm{TiO} / \mathrm{VO}$ opacity is included in the opacity table or not - see Hubeny et al. (2003). The basic conclusions of that paper are the following: The models without $\mathrm{TiO} / \mathrm{VO}$ always exhibit a monotonic temperature/pressure $(T / P)$ profile, with temperature decreasing outward. When the $\mathrm{TiO} / \mathrm{VO}$ opacity is included, the models remain essentially unchanged for larger planet-star separations, simply because TiO and VO are rained out everywhere in the atmosphere, save in the deepest layers which have virtually no effect on emergent spectra. For very close separations, the model with $\mathrm{TiO} / \mathrm{VO}$ exhibits a significant temperature increase toward the surface. In analogy with the solar-system planets, the region of temperature inversion may be called a "stratosphere." But the biggest surprise was that for a certain range of planetary distances, there are actually two legitimate solutions when $\mathrm{TiO} / \mathrm{VO}$ opacity is taken into account - one with a monotonic decrease of temperature toward surface and one exhibiting a stratosphere! 

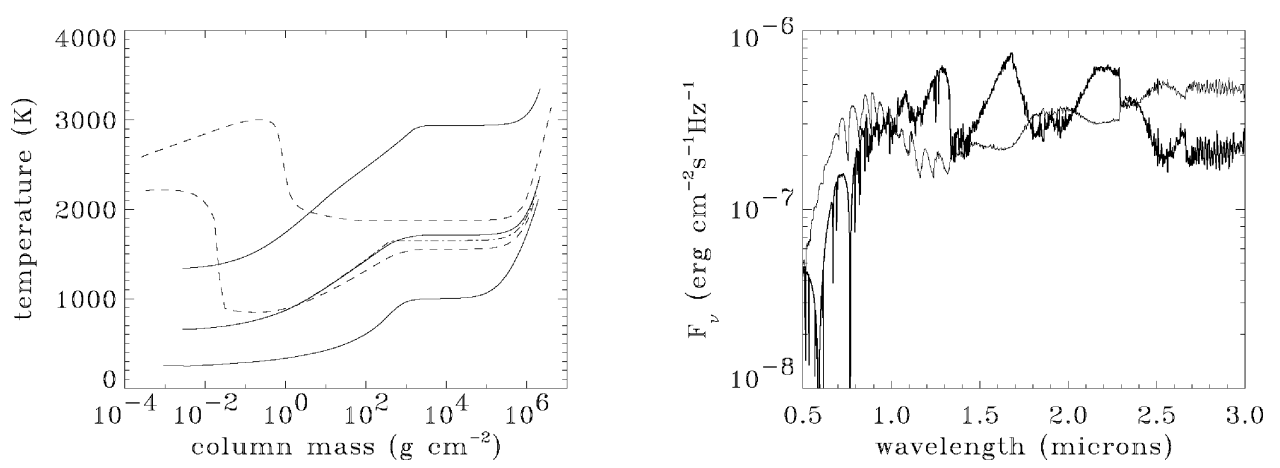

Figure 1. Left panel:Temperature as a function of column mass. Solid lines represent models computed for the opacity table without $\mathrm{TiO} / \mathrm{VO}$, while the dashed and dot-dashed lines represent models with $\mathrm{TiO} / \mathrm{VO}$. The curves represent, from top to bottom, three distances from the central star. The top lines corresponds to $0.0225 \mathrm{AU}$, the middle lines to $0.08 \mathrm{AU}$, and the bottom lines to $0.65 \mathrm{AU}$. The models for the lowest irradiation computed with and without $\mathrm{TiO} / \mathrm{VO}$ are not distinguishable on the plot. The middle dashed and the dot-dashed lines represent the two solutions for the same distance, and thus illustrate the true bifurcation discussed in the text. Right panel: Emergent flux as a function of wavelength for the high-irradiation (distance $0.0225 \mathrm{AU}$ ) model, computed without $\mathrm{TiO} / \mathrm{VO}$ (thick line) and with $\mathrm{TiO} / \mathrm{VO}$ (thin line). The models differ dramatically in the optical and in near-IR region because of the dramatically different surface temperature and the corresponding increase of the $\mathrm{TiO} / \mathrm{VO}$ opacity. (Taken from Hubeny et al. 2003.)

This behavior is illustrated in Fig. 1. Full lines represent models computed for the opacity table without $\mathrm{TiO} / \mathrm{VO}$, while the dashed and dot-dashed lines represent models with $\mathrm{TiO} / \mathrm{VO}$. The middle model exhibits the true bifurcation - the dashed and dotdashed lines correspond to the two solutions for the atmospheric structure with exactly the same input parameters (effective temperature, surface gravity, elemental composition, and irradiation). The fact that the model without $\mathrm{TiO} / \mathrm{VO}$ is very close to the lowsurface-temperature branch of the model with $\mathrm{TiO} / \mathrm{VO}$ clearly indicates that the model bifurcation, and the very existence of the outward temperature increase, arises due to the $\mathrm{TiO} / \mathrm{VO}$ opacity.

The physical explanation of this effect is straightforward - see Hubeny et al. (2003). $\mathrm{TiO} / \mathrm{VO}$ provide a strong opacity in the optical wavelengths, where the external irradiation (in the case of solar-type stars) has its maximum. Therefore, the incoming stellar radiation is efficiently absorbed in the upper atmosphere, thus leading to its heating. The explanation of bifurcation is analogous. An atmosphere either "chooses" a high surface temperature, in which case $\mathrm{TiO} / \mathrm{VO}$ is present, and the incoming stellar flux is efficiently absorbed (so one indeed has, consistently, a high surface temperature). Or, the atmosphere "choses" a low surface temperature, in which case $\mathrm{TiO} / \mathrm{VO}$ does not exist near the surface, and, therefore, there is no efficient mechanism to absorb incoming stellar radiation in the upper atmosphere to heat it.

The difference in the temperature structure is reflected in the emergent spectrum. In the right panel of Fig. 1, we show the emergent flux that coresponds to OGLE-TR-56, with a planet-star separation equal to 0.0225 AU. The thick line is a model without $\mathrm{TiO} / \mathrm{VO}$, and the thin line with $\mathrm{TiO} / \mathrm{VO}$. Similar results were later obtained by Fortney et al. (2006) and Fortney et al. (2008).

However, there is a potentially serious problem with this picture. Since the presence of $\mathrm{TiO}$ and $\mathrm{VO}$ is quite natural at high- $T /$ high- $P$ points, one confronts a situation in which there are two regions rich in $\mathrm{TiO} / \mathrm{VO}$, separated by a middle region that is $\mathrm{TiO} / \mathrm{VO}$ free. 
In a gravitational field with a monotonic pressure profile, this gap could act like a cold trap in which the $\mathrm{TiO} / \mathrm{VO}$ that is transported by molecular or eddy diffusion from the upper low- $P$ region into the intermediate cooler region, would condense and settle out, thereby depleting the upper low- $P \mathrm{TiO} / \mathrm{VO}$-rich region. This would eventually leave no $\mathrm{TiO} / \mathrm{VO}$ at altitude to provide the significant absorption that could lead to a bifurcation.

The situation is much more favorable for very close-in planets, where the temperature is so high that $\mathrm{TiO} / \mathrm{VO}$ is present everywhere in the atmosphere. In this case, one obtains a single solution, that with a stratosphere. We note that the models presented in Hubeny et al. (2003) considered no day/night side heat redistribution. Consequently, these models generally tend to overestimate the atmospheric temperature.

We conclude that $\mathrm{TiO} / \mathrm{VO}$ reliably operate only in a limited temperature range $(T>$ $1500 \mathrm{~K}$ ), because otherwise the cold trap effect may deplete them. One can speculate, though, that diffusion, turbulent mixing, or a wind may actually replenish TiO and VO, but no explicit calculations or even rough estimates have yet been done. Experience from the solar-system giant planets indicates that a number of unknown, optically-active compounds may exist in the upper atmosphere. With extreme irradiation, a complex non-equilibrium photochemistry will very likely take place. Consequently, there may be a number of as yet unknown opacity sources in the upper atmosphere that may build a stratosphere, even in the absence of $\mathrm{TiO} / \mathrm{VO}$. In view of these uncertainties, one is free (indeed, forced) to parameterize the extra opacity; specifically its magnitude $\left(\kappa_{e}\right)$, and its position in the physical and the wavelength space. Therefore, we have adopted such a philosophy. Our approach is described in detail in Burrows et al. (2008).

\section{Day/night side heat redistribution}

In early studies (Sudarsky et al. 2003; Barman et al. 2001; Burrows et al. 2004; Burrows et al. 2006; Burrows et al. 2006), the degree of the day/night side heat redistribution was described through an empirical parameter, originally denoted as $f$. In Burrows et al. (2006) we introduced an analogous parameter, $P_{n}$, as a fraction of incoming flux that is redistributed to the night side. The underlying assumption was that the fraction $P_{n}$ of the incoming flux is somehow removed before the incoming radiation reached the upper boundary of the atmosphere, and deposited at the lower boundary of the night-side atmosphere.

Although this treatment is useful for obtaining a rough estimate of the effects of day/night side heat redistribution, it is unsatisfactory from the physical point of view. There are two main problems: (i) it is unphysical to assume that the incoming flux is removed at the top of the day-side atmosphere and injected in the bottom of the nightside atmosphere, and (ii) the calculated entropies in the convection zones at the day and the night side may be generally different. Since convective transport is very efficient, the temperature gradient is essentially exactly adiabatic. Consequently, the whole convective core has to be isentropic, and, thus, cannot exhibit any difference between the day and night sides.

To overcome these problems, we have recently adopted an improved, albeit still parametric, approach - Burrows et al. (2008). Out of the total incoming energy, the fraction $\left(1-P_{n}\right)$ is assumed to be radiated on the day side, and the fraction $P_{n}$ on the night side. The irradiation energy is removed on the day side in a given depth range, parameterized by limiting pressures $P_{0}$ and $P_{1}$. This energy is deposited on the night side in depth range parameterized by limiting pressures $P_{0}^{\prime}$ and $P_{1}^{\prime}$, which are generally different from $P_{0}$ and $P_{1}$, but in actual calculations we usually take them to coincide with $P_{0}$ and $P_{1}$. The modeling proceeds as follows: at the day side, the input parameters are $\log g$ ( $g$ being 
the surface gravity); $T_{\text {int }}$ (which corresponds to $T_{\text {eff }}$ in the case of stellar atmospheres); $P_{n} ; P_{0}$; and $P_{1}$. Other parameters are the distance and the radius of the parent star, and the elemental composition (which is assumed to be the same on the night side). The computed model gives the structure (essentially, the $T / P$ profile and the self-consistent radiation intensity), plus the entropy in the convection zone. At the night side, the input parameters are: $\log g$ (which must be the same as at the day side); $P_{n}$; limiting pressures $P_{0}^{\prime}$ and $P_{1}^{\prime}$; and a trial $T_{\text {int }}^{\text {night }}$. The model again yields a $T / P$ profile, and the entropy at the convection zone, which is generally different from the entropy at the day side. The intrinsic temperature $T_{\mathrm{int}}^{\mathrm{night}}$ is adjusted by trial and error so that both entropies (day and night) match. We note that with strong irradiation, the convection zone may be very deep (at $\tau_{\text {ross }}$ of the order of $10^{4}$ to $10^{6}$ ), and, therefore, the entropy matching is important for evolutionary models, and not so much for predicting emergent spectra.

\section{Theoretical interpretation of the secondary eclipse spectra}

For close-in giant planets the planet-star contrast ratios in the mid-infrared are relatively large, often exceeding $10^{-3}$, and such contrasts are well within reach of the infrared space telescope Spitzer. Using its IRAC and MIPS cameras, and the IRS spectrometer, one can now measure the summed light of the planet and the star in and out of the secondary eclipse, and, from the difference, determine the planet's spectrum at superior conjunction (see several reviews in these Proceedings). This has led to a breakthrough in the study of extrasolar planets, for it provides a means to probe the physics, chemistry, and even meteorology of their atmospheres.

Before this conference, secondary-eclipse fluxes in the IRAC and MIPS channels had been measured for five transiting planets - HD189733b, TrES-1, HD 209458b, HD 149026b, and GJ 436b. Our group has performed a detailed analysis of four of them (Burrows et al. 2008). So far, we have not analyzed GJ 436b.

The results are displayed in the four panels of Fig. 2 for the four transiting planets. For HD 209458b, we show three models with an upper-atmosphere extra absorber (and with resulting stratospheres), and one traditional model without an extra absorber. The values of the redistribution parameter $P_{n}$ for the models with an extra absorber are 0.3 , 0.4 , and 0.5 , and the limiting pressures for the energy sink are 0.01 and 0.1 bars. The extra absorber, assumed to contribute between 0.4 and $1 \mu \mathrm{m}$, is placed at altitude below pressures of 25 mbars. The corresponding $T / P$ profiles for the four HD 209458b models depicted in Fig. 5, together with the "formation depths," defined as the position in the atmosphere where the monochromatic optical depth reaches $2 / 3$, are displayed in Fig. 3 . The plot explains why the flux in the IRAC 2, 3, 4, and MIPS bands are higher for the model with an extra absorber (stratosphere), while it is lower in the IRAC 1 band.

Figure 2 shows that for HD209458b the models with a stratosphere fit the IRAC 1, 2, and 4 points quite well. However, the IRAC 3 point near $5.8 \mu \mathrm{m}$ is difficult to fit since the radiation in this band is formed at essentially the same position in the atmosphere as the IRAC 4. Analogously, for HD149026b, the model with a stratosphere is clearly preferable. The situation for HD $189733 \mathrm{~b}$ is uncertain; in any case a stratosphere may exist, but there is no compelling case for it. On the other hand, the TrES-1 data are fitted perfectly with a traditional model without a stratosphere. Since the total irradiated flux is largest for HD 149026b and smallest for TrES-1 - see Burrows et al. (2008), the presence of the stratosphere seems to be very roughly correlated with the total stellar flux at the planet's surface. In Burrows et al. (2008), we have analyzed also the observed light curves of two non-transiting planets ( $v$ And b, and HD179949b), and found that for $v$ And b the presence of a stratosphere is strongly indicated in order to fit the observed data. 

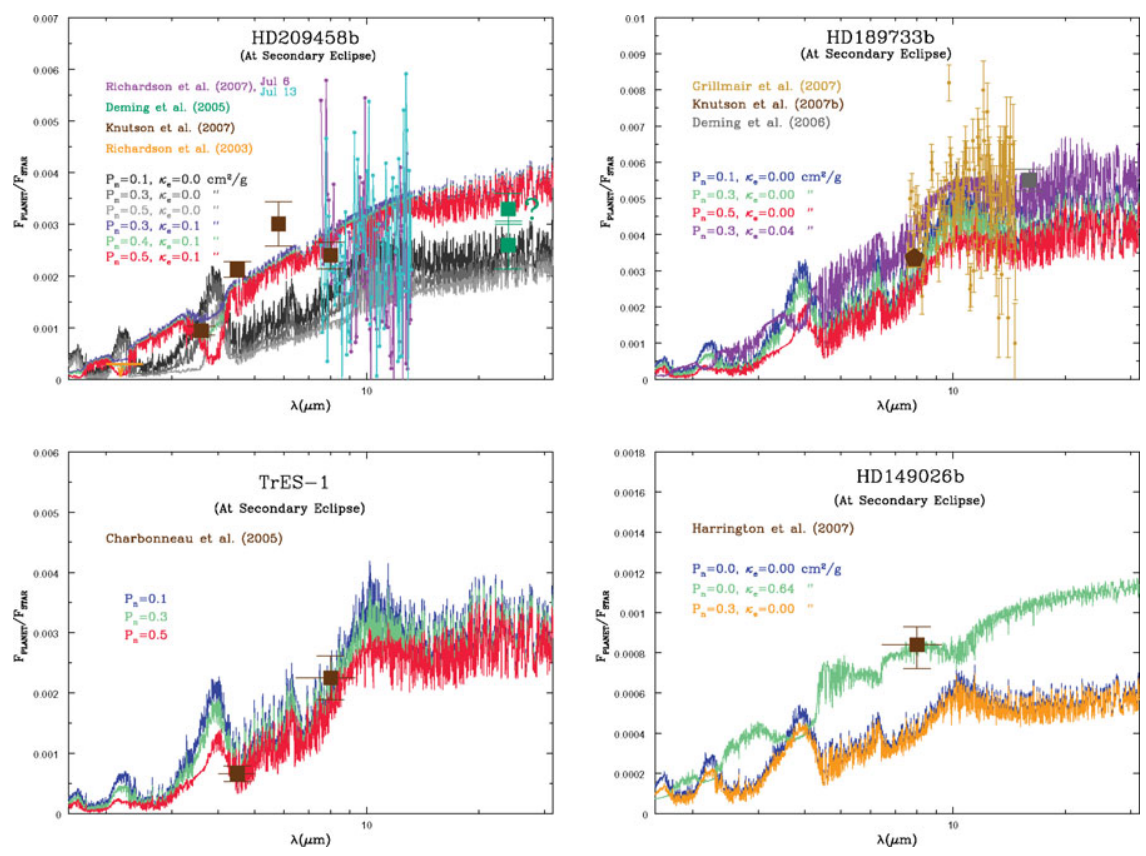

Figure 2. The planet/star flux ratios versus wavelength for various models of four transiting planets measured by Spitzer at secondary eclipse. Notice the different scales employed in each panel. Models for different values of $\mathrm{P}_{n}$ and $\kappa_{\mathrm{e}}$ are provided where appropriate. On the upper-left panel (HD 209458b), models with the lighter gray shade(s) are for the higher value(s) of $\mathrm{P}_{n}$. Notice also that two different values for the flux at $24 \mu \mathrm{m}$ are shown on this same panel. The one with the question mark is a tentative update to the Deming et al. (2005) 24- $\mu \mathrm{m}$ measurement, kindly provided by Drake Deming (private communication). If the flux at $24 \mu \mathrm{m}$ is indeed $\sim 0.0033 \pm 0.0003$, then our model(s) with inversions provide the best fit at that wavelength as well. (Taken from Burrows et al. 2008.)

\section{Conclusions}

We have found (Burrows et al. 2007; Burrows et al. 2008) that a consistent fit to all data at secondary eclipse for several strongly irradiated transiting planets (HD 209458b, HD 149026b, and possibly HD 189733b), and very likely a non-transiting planet $v$ And b, requires that their atmospheres have temperature inversions - stratospheres - at altitude. Such a thermal inversion affects: (i) planet/star contrast ratios at the secondary eclipse; (ii) their wavelength dependences; and (iii) day-night flux contrasts during a planetary orbit. Moreover, the presence of the thermal inversion/stratosphere seems to roughly correlate with the total irradiated flux.

Temperature inversion is caused either by $\mathrm{TiO} / \mathrm{VO}$, as first suggested by Hubeny et al. (2003), or by another, as yet unidentified, opacity sources. These may be tholins, polyacetylenes, or various non-equilibrium compounds. We invoke such extra absorbers, because a cold-trap effect can operate to deplete the upper atmosphere of $\mathrm{TiO} / \mathrm{VO}$. However, one may speculate that with ongoing mass loss and/or rotational shear instabilities the atmosphere may be partially replenished in $\mathrm{TiO} / \mathrm{VO}$. Therefore, while $\mathrm{TiO} / \mathrm{VO}$ might be responsible for the formation of thermal inversions in the strongly irradiated planets, the exact nature of the absorber must be viewed as very uncertain. 


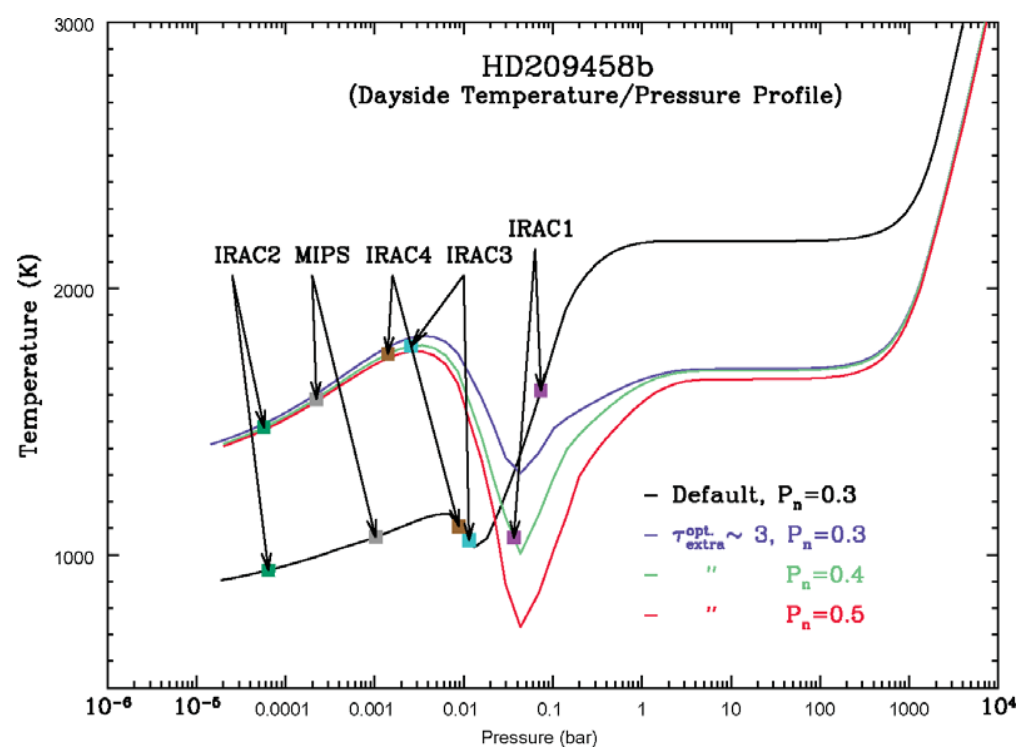

Figure 3. Day-side temperature/pressure profiles for the four models for HD209458b depicted in the upper left panel of Fig. 5. We also indicate the position where the monochromatic optical depths for the characteristic wavelengths of the four IRAC and the MIPS channel are equal to 2/3. (Taken from Burrows et al. 2007).

\section{References}

Barman, T., Allard, F., \& Hauschildt, P. (2001) ApJ, 556, 885

Burrows, A. et al. 1997, ApJ 491, 875

Burrows, A. \& Sharp, C. 1999, ApJ 512, 843

Burrows, A., Sudarsky, D., \& Hubeny, I. 2004, ApJ, 609, 407

Burrows, A., Hubeny, I., \& Sudarsky, D. 2005, ApJ, 625, L135

Burrows, A., Sudarsky, D., \& Hubeny, I. 2006, ApJ, 650, 1140

Burrows, A., Hubeny, I, Budaj, J., Knutson, H. A., \& Charbonneau, D. 2007, ApJ, 668, L171

Burrows, A., Budaj, J. \& Hubeny, I. 2008a, ApJ, 678, 1436

Charbonneau, D. et al. 2005, ApJ, 626, 523

Deming, D., Seager, S., Richardson, L. J., \& Harrington, J. 2005, Nature, 434, 740

Deming, D., Harrington, L. J., Seager, S., Richardson, L. J. 2006, ApJ, 644, 560

Fortney, J. J., Saumon, D., Marley, M. S., Lodders, K., \& Freedman, R. S. 2006, ApJ, 642, 495

Fortney, J. J., Lodders, K., Marley, M. S., \& Freedman, R. S. 2008, ApJ, 678, 1419

Harrington, L. J. et al. 2007, Nature, 447, 691

Hubeny, I. 1988, Computer Phys. Commun., 52, 103

Hubeny, I. \& Lanz, T. 1995 ApJ, 439, 875

Hubeny, I., Burrows, A., \& Sudarsky, D. 2003, ApJ, 594, 1011

Knutson, H. A., Charbonneau, D., Noyes, R. W., Brown, T. M., Gilliland, R. L. 2007, ApJ, 655, 564

Knutson, H. A., Charbonneau, D., Allen, L. E., Burrows, A., Megeath, S. T. 2008 ApJ, 673, 526

Konacki, M., Torres, G., Jha, S., \& Sasselov, D. 2003, Nature, 421, 507

Richardson, L. J., Deming, D., \& Seager, S. 2003, ApJ, 597, 581

Richardson, L. J., Deming, D., Horning, K., Seager, S., \& Harrington, J. 2007, Nature, 445, 892

Seager, S. \& Sasselov, D. 1998 ApJ, 502, L157

Sharp, C. \& Burrows, A. 2007 ApJS, 168, 140

Sudarsky, Burrows, A., \& Hubeny, I. 2003, ApJ, 588, 1121

Sudarsky, Burrows, A., \& Pinto, P. 2000, ApJ, 538, 885 